\title{
Kinematic and Dynamic Simulation of the Wheel Loaders with the Z-bar Working Mechanism
}

\author{
Dragoslav Janošević, ${ }^{*}{ }^{*}$ Jovan Pavlović ${ }^{1}$, Vesna Jovanović ${ }^{1}$, Predrag Milić ${ }^{1}$ \\ ${ }^{1}$ University of Niš, Faculty of Mechanical Engineering
}

This paper presents the mathematical model and program for the kinematic and dynamic analysis of wheel loader with the Z-bar working mechanism. Kinematic analysis determined a linear and angular speed and acceleration of centar of mass for the kinematic chain members. Dynamic analysis determined an inertial force and rotational inertia torque of centar of mass for the members of kinematic chain of the machine by Newton-Euler dynamic equations. As an example, the results of kinematic and dynamic analysis are given for the wheel loader which mass is $15000 \mathrm{~kg}$ and volume of bucket is $2.7 \mathrm{~m}^{3}$.

Keywords: Wheel loaders, kinematic and dynamic analysis

\section{INTRODUCTION}

The wheel loaders are characterized by the possibility of performing different functions due to different configurations of the working mechanism and the possibility of equipping with various tools.

The main (primary) function of the loader is cyclic (intermittent) transport of a bulk material at a certain distance (up to $l=50 \mathrm{~m}$ ). In general, the basic function of the loader consists of the following partial functions: loading, transport, unloading the materials and return again to a new place of loading.

In this paper is observed general configuration of kinematic chain, that is consists of: rear $L_{1}$ (Fig. 1) and front $L_{2}$ supporting-moving mechanism and the manipulator with $\operatorname{arm} L_{3}$ and bucket $L_{4}$. With direct link of pairs of arm hydro-cylinders $c_{3}$ for the frame of moving mechanism $L_{2}$ and for $\operatorname{arm} L_{3}$, is formed drive mechanism of arm.

The drive mechanism of bucket is consists of one or two hydro-cylinders $c_{4}$ which are on one side connected with direct link to the frame of moving mechanism $L_{2}$, and from other side is connected, via two-arm lever $c_{41}$ and rod $c_{5}$, to bucket $L_{4}$, thus formed configuration in shape of letter " $Z$ " by which the kinematics of the manipulator is named.

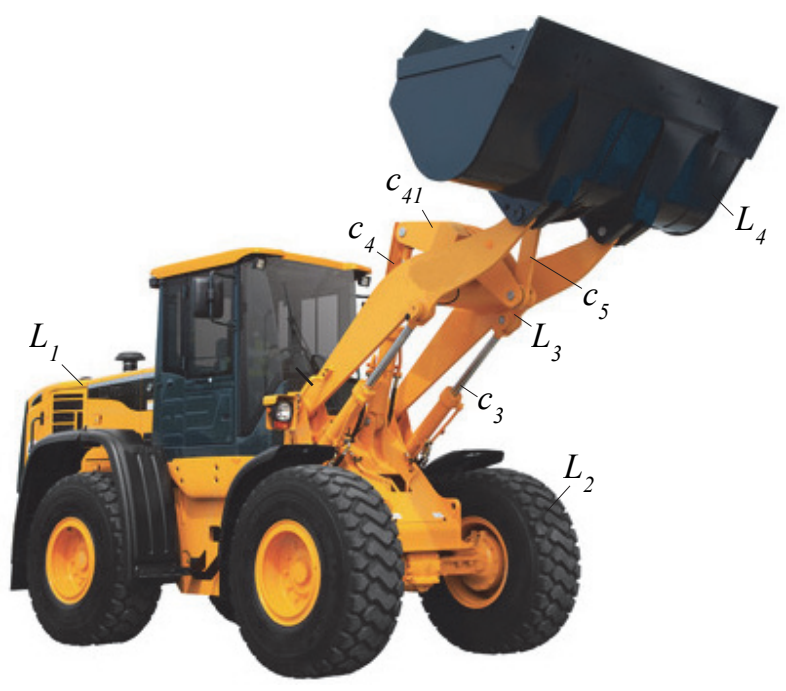

Fig.1. The physical model of wheel loader

\section{MATHEMATICAL MODEL}

The mathematical model of loader with the Z-bar kinematics is defined to allow the numerical simulation of loader's manipulative task in function of cycle duration. The mathematical model also determine the kinematic and dynamic parameters of the kinematic chain and drive mechanism of machine[1].

The simulation starts from a set of given values:

$$
P_{u}=\left\{V, b_{4}, Y_{i}, \alpha_{i}, t_{c}, K_{z}\right\}
$$

where is: $V$ - volume of bucket, $b_{4}$ - width of bucket, $Y_{i}$ - unloading height, $\alpha_{i}$ - bucket unloading angle, $t_{c}$ - time of manipulative task, $K_{z}$ - subset of the characteristics of the material.

Defined mathematical model of the loader kinematic chain is based on general theorems of mechanics. The members of the loader kinematic chain builds kinematic pairs of fifth class - rotary joint with one degree of freedom. An axes of joints are axes of relative rotation of the members who build the kinematic chain pairs.

The member of loader kinematic chain $L_{i}$ is determined in its local coordinate system $O_{i} x_{i} y_{i} z_{i}$ with set of a geometric and dynamic sizes:

$$
L_{i}=\left\{\widetilde{\boldsymbol{e}}_{i}, \widetilde{\boldsymbol{s}}_{i}, \widetilde{\boldsymbol{t}}_{i}, m_{i}, \widetilde{J}_{i}\right\}
$$

where is: - $\widetilde{\boldsymbol{e}}_{i}$ - orientation of an axis of joint $O_{i}$ with whom the member $L_{i}$ is connected to previous member $L_{i-1}, \widetilde{\boldsymbol{s}}_{i}$ position vector of the joint center with whom the member $L_{i}$ is conected to forward member $L_{i+1}$, intensity of vector $\boldsymbol{s}_{i}$ represents the kinematic length of member, $\tilde{\boldsymbol{t}}_{i}$ - position vector of center of mass $m_{l}$ of member chain $L_{i}$ in absolute coordinate system, $\widetilde{J}_{i}$ - moment of inertia tensor of member.

Values that is tagged with "wave" above the mark, are defined in the local coordinate system of members.

\subsection{Kinematic model}

The mathematical model of kinematic chain corresponds to the next inner (generalized) coordinates: 


$$
\theta_{i}=\left\{\theta_{1}, \theta_{3}, \theta_{4}\right\}
$$

where is: $\theta_{1}=x_{1}$ - penetration lenght of bucket, apropos the relative movement of the loader in relation to the surface, $\theta_{3}$ - relative angle position of arm $L_{3}$ relative to the $\mathrm{x}$ - axis of the previous member $L_{2}, \theta_{4}$ - relative angle of the bucket $L_{4}$ relative to the $\mathrm{x}$ - axis of the previous member $L_{3}$.

The generalized coordinates determine position of the members of kinematic chain $L_{3}$ and $L_{4}$, relative to an absolute coordinate system, and they are:

$$
\begin{gathered}
\varphi_{3}=\theta_{3} \\
\varphi_{4}=\theta_{3}+\theta_{4}
\end{gathered}
$$

The vectors of: position of the center of the joint $\boldsymbol{r}_{i}$, the center of mass $\boldsymbol{r}_{t i}$ of kinematic chain members and the center of the cutting edge of the bucket $\boldsymbol{r}_{w}$, relative to an absolute coordinate system are determined by the equations:

$$
\begin{aligned}
& \boldsymbol{r}_{i}=\sum_{j=l}^{i-l} A_{j o} \widetilde{\boldsymbol{s}}_{j} \\
& \boldsymbol{r}_{w}=\sum_{j=l}^{4} A_{j o} \widetilde{\boldsymbol{s}}_{j} \\
& \boldsymbol{r}_{t i}=\boldsymbol{r}_{i}+A_{i o} \tilde{\boldsymbol{t}}_{i}
\end{aligned}
$$

where is: $A_{j o}, A_{i o}$ - transformation matrix for the transformation of a vector from the local to the absolute coordinate system [3].

The transformation matrix of mathematical model:

$$
\begin{aligned}
A_{3 o} & =\left|\begin{array}{ccc}
\cos \varphi_{3} & -\sin \varphi_{3} & 0 \\
\sin \varphi_{3} & \cos \varphi_{3} & 0 \\
0 & 0 & 1
\end{array}\right| \\
A_{4 o} & =\left|\begin{array}{ccc}
\cos \varphi_{4} & -\sin \varphi_{4} & 0 \\
\sin \varphi_{4} & \cos \varphi_{4} & 0 \\
0 & 0 & 1
\end{array}\right|
\end{aligned}
$$

The work of loader is characteristic in that the manipulative tasks are numerous and cyclic but with the

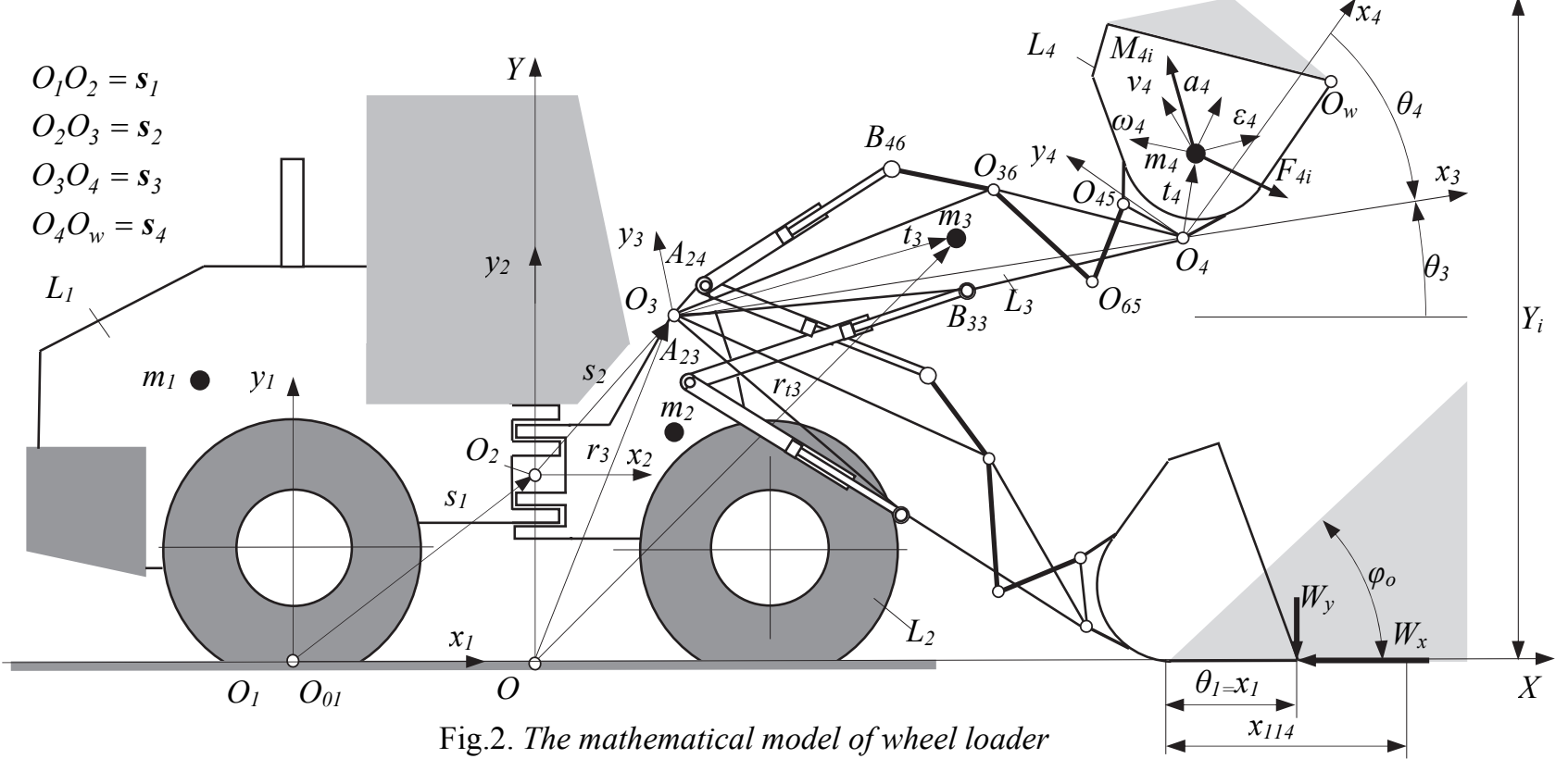

Janošević, D. - Pavlović, J. - Jovanović, V. - Milić, P.

same operations structure. However, at each cycle, a operation have different parameters. For ease defining, each parameter of the manipulative task is marked with index $i j$, where index $i$ denotes the number of the kinematic chain member that carrier operations with the index $j$.

The manipulative task that is analyzed in this paper consists of the following operations:

- loading the material with bucket $(j=1)$,

- lifting the material to a certain height $(j=2)$, and

- unloading the bucket $(j=3)$.

The inner coordinates of operations $j$ of manipulative task corresponds to a specific range of motion of each kinematic chain member $L_{i}$ :

$$
\delta_{i j}=\theta_{i j 4}-\theta_{i j 1}
$$

where is: $\delta_{i j}$ - movement range of the members $i$ and the operation $j, \theta_{i j 1}, \theta_{i j 4}$ - inner coordinates of the member position $L_{i}$, on start $k=1$ and on end $k=4$ of operation $j$.

For each member $L_{i}$ of the kinematic chain and for each operation $j$ the phase time are given: a) time $t_{i j l}$ of beginning of the operation and the start of acceleration, $b$ ) end time $t_{i j 2}$ of acceleration, c) beginning time $t_{i j 3}$ of deceleration, d) end time $t_{i j 4}$ of operation and end of deceleration. As an example, the inner coordinates of the members $L_{1}$ and $L_{2}$ at the operation of loading material $(j=1)$ are:

$$
x_{11}=\left\{\begin{array}{l}
x_{111}=0 \forall t=t_{111} \\
x_{114}=\left(\frac{2 V}{b_{4} \cdot \operatorname{tg} \varphi_{o}}\right)^{0,5} \forall t=t_{114}
\end{array}\right.
$$

Where is: $\varphi_{o^{-}}$natural angle of material slope.

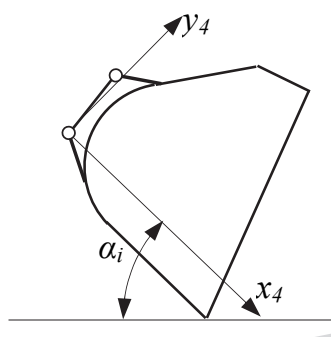


The members of the loader's kinematic chain, which are carriers of the certain operations of manipulative task with a known range of motion, given phase times and adopted cycloid change of motion, corresponds to a certain maximum speeds (Fig. 3):

$$
\dot{\theta}_{i j m}=\frac{2 \delta_{i j}}{\left(t_{i j 3}+t_{i j 4}\right)-\left(t_{i j 1}+t_{i j 2}\right)}
$$

on the basis of which are determined change of the speed:

$$
\dot{\theta}_{i j}=\left\{\begin{array}{l}
\frac{\dot{\theta}_{i j m}}{2}\left[1-\cos \left(\frac{t-t_{i j 1}}{t_{i j 2}-t_{i j 1}}\right) \pi\right] \forall t_{i j 1} \leq t \leq t_{i j 2} \\
\dot{\theta}_{i j m} \forall t_{i j 2} \leq t \leq t_{i j 3} \\
\frac{\dot{\theta}_{i j m}}{2}\left[1+\cos \left(\frac{t-t_{i j 3}}{t_{i j 4}-t_{i j 3}}\right) \pi\right] \forall t_{i j 3} \leq t \leq t_{i j 4}
\end{array}\right.
$$

and acceleration:

$$
\ddot{\theta}_{i j}=\left\{\begin{array}{l}
\frac{\dot{\theta}_{i j m}}{2} \frac{\pi}{t_{i j 2}-t_{i j 1}} \sin \left(\frac{t-t_{i j 1}}{t_{i j 2}-t_{i j 1}}\right) \pi \forall t_{i j 1} \leq t \leq t_{i j 2} \\
0 \forall t_{i j 2} \leq t \leq t_{i j 3} \\
-\frac{\dot{\theta}_{i j m}}{2} \frac{\pi}{t_{i j 4}-t_{i j 3}} \sin \left(\frac{t-t_{i j 3}}{t_{i j 4}-t_{i j 3}}\right) \pi \forall t_{i j 3} \leq t \leq t_{i j 4}
\end{array}\right.
$$

The kinematic values of the kinematic chain members of loaders are: linear $v_{i}$ and angular velocity $\omega_{i}$ and linear $a_{i}$ and angular accelerations $\varepsilon_{i}$ of chain member $L_{i}$, and they are determine relative to an absolute coordinate system with recursive equations [2][3]:

$$
\begin{gathered}
\omega_{i}=\omega_{i-1}+\dot{\theta}_{i} \boldsymbol{e}_{i} \\
\boldsymbol{\varepsilon}_{i}=\boldsymbol{\varepsilon}_{i-1}+\ddot{\theta}_{i} \boldsymbol{e}_{i}+\dot{\theta}_{i}\left(\omega_{i} \times \boldsymbol{e}_{i}\right) \\
\boldsymbol{v}_{i}=\boldsymbol{v}_{i-1}-\boldsymbol{\omega}_{i-1} \times \boldsymbol{r}_{i-1, i}+\omega_{i} \times \boldsymbol{r}_{i, i+1}+\dot{\theta}_{i} \boldsymbol{e}_{i} \\
\boldsymbol{a}_{i}=\boldsymbol{a}_{i-1}-\boldsymbol{\varepsilon}_{i-1} \times \boldsymbol{r}_{i-1, i}-\omega_{i-1} \times\left(\omega_{i-1} \times \boldsymbol{r}_{i-1, i}\right)+ \\
+\boldsymbol{\varepsilon}_{i} \times \boldsymbol{r}_{i, i+1}+\boldsymbol{\omega}_{i} \times\left(\omega_{i} \times \boldsymbol{r}_{i, i+1}\right)+\ddot{\theta}_{i} \boldsymbol{e}_{i}+2 \dot{\theta}_{i}\left(\omega_{i} \times \boldsymbol{\varepsilon}_{i}\right)
\end{gathered}
$$

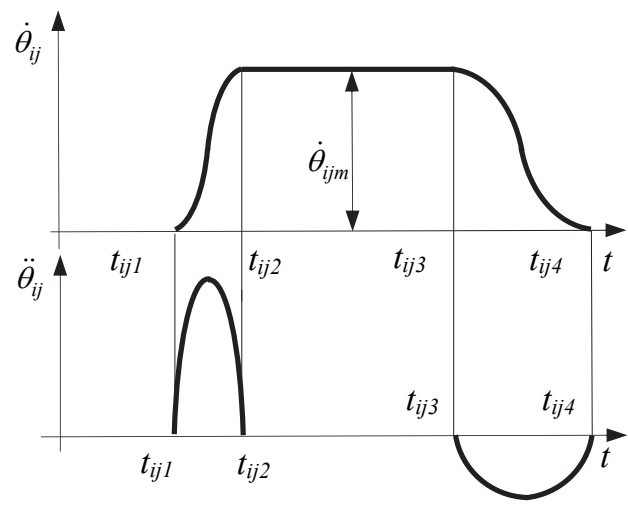

Fig. 3. Trapezoidal model of motion of the kinematic chain members with cyclical changes of the angular velocity

\subsection{Dynamic model}

On the members of the loader's kinematic chain, during cycle operation, act internal (gravitational, inertial) and external (technological) loads.

Dynamic values of the member $L_{i}$ are: inertial force $F_{i}$ which is determined by second Newton's law:

$$
\boldsymbol{F}_{i}=-m_{i} \boldsymbol{w}_{i}
$$

and rotational inertia torque $M_{i}$, which is determined based on Euler's dynamic equations:

$$
\widetilde{\boldsymbol{M}}_{i}=-\widetilde{J}_{i} \widetilde{\boldsymbol{\varepsilon}}_{i}+\widetilde{\boldsymbol{\omega}}_{i} \times \widetilde{J}_{i} \widetilde{\boldsymbol{\omega}}_{i} \quad \boldsymbol{M}_{i}=A_{i o} \widetilde{\boldsymbol{M}}_{i}
$$

where is: $\widetilde{\boldsymbol{\omega}}_{i}=A_{o i} \boldsymbol{\omega}_{i}, \quad \widetilde{\varepsilon}_{i}=A_{o i} \varepsilon_{i}$ - angular velocity and angular accelerations of the member $L_{i}$ in local coordinate system $O_{i} x_{i} y_{i} z_{i}, A_{o i}$ - transformation matrix of the model for transformation of the vector from absolute to local coordinate system

The total force related to the center of mass of member $L_{i}$, taking the influence of gravity, is equal to:

$$
\boldsymbol{F}_{u i}=\boldsymbol{F}_{i}+m_{i} \boldsymbol{g}
$$

On separated kinematic chain member $L_{i}$ (Fig.2) besides the total force $F_{u i}$ and torque $M_{i u}$ is acting also reactions $-F_{r i+1},-M_{r i-1}$ of forward member $L_{i+1}$ in joint $O_{i+1}$ and force $F_{r i}$ and torque $M_{r i}$ in joint $O_{i}$. From the equilibrium conditions of separated member $L_{i}$ it can be determined force $F_{r i}$ and torque $M_{r i}$ in joint $O_{i}$ :

$$
\begin{gathered}
\boldsymbol{F}_{r i}=\boldsymbol{F}_{c i}+\boldsymbol{W}+\sum_{j=i}^{4} \boldsymbol{F}_{u j} \forall i=3,4 \\
\boldsymbol{M}_{r i}=\boldsymbol{M}_{c i}+\left(\boldsymbol{r}_{w}-\boldsymbol{r}_{i}\right) \times \boldsymbol{W}+\sum_{j=i}^{4} \boldsymbol{M}_{j}+\sum_{j=i}^{4}\left(\boldsymbol{r}_{t j}-\boldsymbol{r}_{i}\right) \times \boldsymbol{F}_{u j} \forall i=3,4
\end{gathered}
$$

where is: $\boldsymbol{F}_{c i}$ - force of the hydro-cylinder or two-arm lever of the drive mechanism of member $L_{i}, \boldsymbol{W}$ - loading resistances (digging) which is acting in center of the bucket cutting edge $O_{w}$ and $\boldsymbol{M}_{c i}$ - torque of drive mechanism for joint $O_{i}$. Dynamic torque of drive mechanism are:

$$
\boldsymbol{M}_{o i}=\boldsymbol{M}_{r i} \cdot \boldsymbol{e}_{i}
$$

\subsubsection{Digging resistances.}

The external (technological) loads during operation of material loading, occurs in the form of loading resistances (digging) of material. The material loading operations is usually performed thus the bucket first penetrate into a pile of material by movement of the loader, and then comes to filling the bucket by rotation of bucket around the arm top.In horizontal penetration of the bucket when loader's speed is $v$ (Fig. 4), there are the following components of loading resistance (digging): resistances along the horizontal cutting edge $W_{x l}$, resistances along the vertical cutting edge $W_{x 2}$, the frictional resistances of the affected material on the bottom of the bucket $W_{x 3}$, resistances to friction of inner and outer sides of the bucket $W_{x 4}$, the frictional resistance of the outer surface of the bottom of the bucket $W_{x 5}$, friction resistance of material on the bottom of the bent part of bucket $W_{x 6}$. Resistance $W_{x l}$ along the horizontal cutting edge [7]: 


$$
W_{x l}=k_{x} \cdot b_{4} \cdot x_{1}
$$

where is: $k_{x}$ - specific resistance of the materials at bucket penetration $\left[\mathrm{kN} / \mathrm{m}^{2}\right], x_{1}$ - current distance from the top of the bucket cutting edge to the bottom of material pile (length of penetration) $[\mathrm{m}], W_{x l}$ - resistance $[\mathrm{kN}]$.

Resistances $W_{x 2}$ along the vertical cutting edge:

$$
W_{x 2}=\frac{1}{2} k_{x} \cdot h \cdot x_{1}=\frac{1}{2} k_{x} \frac{\operatorname{tg} \varphi_{o} \cdot \operatorname{tg} \beta}{\operatorname{tg} \varphi_{o}+\operatorname{tg} \beta} \cdot x_{1}^{2}
$$

where is: $h$ - slice thickness, $\beta$ - tip angle of bucket side profile.

The current slice thickness $h$ of the material can be determined from equation (Fig. 4a):

from which follows:

$$
h=e \cdot \operatorname{tg} \beta=\left(x_{1}-e\right) \operatorname{tg} \varphi_{o}
$$

$$
e=\frac{x_{1} \cdot \operatorname{tg} \varphi_{o}}{\operatorname{tg} \varphi_{o}+\operatorname{tg} \beta}
$$

so when the values of $e$ is transform in equation 27 we have:

$$
h=\frac{x_{1} \cdot \operatorname{tg} \varphi_{o} \cdot \operatorname{tg} \beta}{\operatorname{tg} \varphi_{o}+\operatorname{tg} \beta}
$$

The resistances $W_{x 3}$ of frictional of the affected material on the bottom of the bucket:

$$
W_{x 3}=\mu_{2} \iint_{A_{d}} p_{d} \cdot d A_{d}
$$

where is: $\mu_{2}$ - coefficient of material friction on the metal, $p_{d}$ - the pressure of the affected material to the surface $A_{d}$ of the bottom part of the bucke.

Pressure $p_{d}$ at the elementary surface $d A_{d}=b_{5} \cdot d x$ have value (Fig.4c):

$$
p_{d}=g \rho_{z} x_{1} \cdot \operatorname{tg} \varphi_{o}\left(1+\sin ^{2} \varphi_{o}\right)
$$

Substitution of expressions for $p_{d}$ and $d A_{d}$ in equation (2.48) we get:

$$
W_{x 3}=\frac{1}{2} \mu_{2} \cdot b_{4} \cdot g \rho_{z} \cdot \operatorname{tg} \varphi_{o}\left(1+\sin ^{2} \varphi_{o}\right) x_{1}
$$

where is: $\rho_{z}$ - density of the affected materials $\left[\mathrm{kg} / \mathrm{m}^{3}\right], b_{5}-$ bucket width $[m], W_{x 3}$ - resistance $[N]$.

The frictional resistance $W_{x 4}$ of the affected materials on the inner surface of the side sheet metal of bucket:

$$
W_{x 4}=\mu_{2} \iint_{A_{b}} p_{b} \cdot d A_{b}=\mu_{2} \cdot p_{b 5} \cdot A_{b}
$$

where is: $p_{b}$ - lateral pressure of the affected materials on the elementary side surface $d A_{b}$ which covers the affected material inside the bucket, $p_{b s}$ - medium pressure to the total lateral surface $A_{b}$, which covers the affected material.

Medium pressure $p_{b s}$ corresponding one third of the material pressure $p_{b o}$ in point $O_{w}$ (Fig. 4a):

$$
p_{b s}=\frac{1}{3} p_{b o}=\frac{1}{3} g \rho_{z} \cdot x_{1} \cdot \operatorname{tg} \varphi_{o}\left(1+\sin ^{2} \varphi_{o}\right) \cdot \operatorname{tg}^{2}\left(\frac{\pi}{4}-\frac{\rho}{2}\right)
$$

Where is: $\rho$ - internal angle of friction of materials.
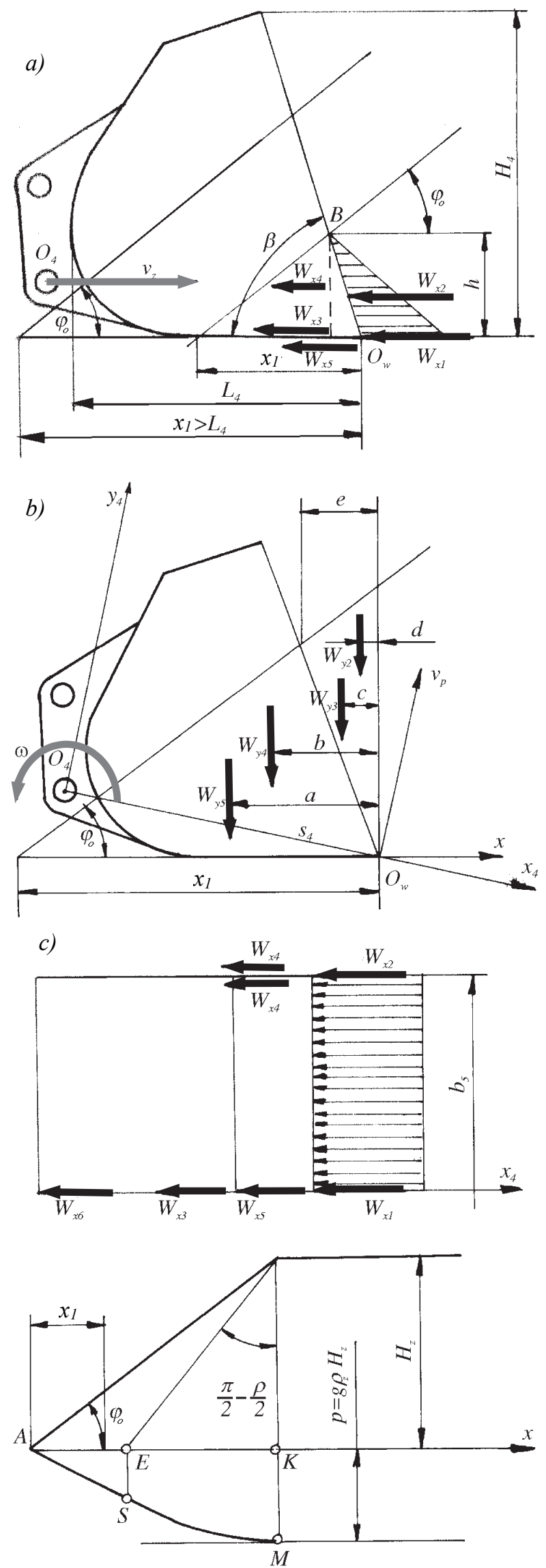

Fig.4. Digging resistance of bucket: a) components of resistance at bucket penetration, b) components of resistance at bucket filling, c) pressure distribution of material on the bucket internal side 
Total current lateral surface:

$$
A_{b}=\frac{1}{2} x_{1} \cdot h=\frac{\operatorname{tg} \varphi_{o} \cdot \operatorname{tg} \beta}{2\left(\operatorname{tg} \varphi_{o}+\operatorname{tg} \beta\right)} x_{1}^{2}
$$

Substitution of equations (35) and (36) into equation (34) it is obtained resistance $W_{x 4}$ :

$$
W_{x 4}=\mu_{2} \frac{g \cdot \rho_{z} \cdot \operatorname{tg} \beta \cdot \operatorname{tg}^{2} \varphi_{o}\left|\left(1+\sin ^{2} \varphi_{o}\right) \cdot \operatorname{tg}^{2}\left(\frac{\pi}{4}-\frac{\rho}{2}\right)\right|}{6\left(\operatorname{tg} \varphi_{o}+\operatorname{tg} \beta\right)} x_{1}^{3}
$$

The frictional resistance $W_{x 5}$ of the outer surface of the bottom of the bucket on material is equal:

$$
W_{x 5}=W_{x 3}
$$

If at penetration the bucket rely with its weight on the surface. In the case of bucket penetration, when arm is carring the bucket this resistance does not exist:

$$
W_{x 5}=0
$$

The resistance $W_{x 6}$ of material friction on the bottom of the folded portion of the bucket occurs if $x_{1}>L_{4}$ :

$$
W_{x 6}=2 \cdot g \rho_{z} \cdot b_{4} \cdot \operatorname{tg} \varphi_{o} \cdot \operatorname{tg}^{2}\left(\frac{\pi}{4}+\frac{\varphi_{o}}{2}\right) \cdot\left(x_{1}-L_{4}\right)^{2}
$$

where is: $L_{4}$ - the distance from the tip of the cutting edge to the bottom of the bucket.

The total resistance $W_{x}$ of bucket penetration:

$$
W_{x}=W_{x 1}+2 W_{x 2}+W_{x 3}+4 W_{x 4}+W_{x 5}+W_{x 6}
$$

At the end of the penetration, by turning the bucket around the arm top, with angular velocity $\omega$ (Fig.4b) it comes to the bucket filling while it occures following vertical resistance: slippage resistance of the affected material's prisms on the frontal plane of massif $W_{y l}$, slippage resistance of affected material prism to the vertical side of massif $W_{y 2}$, penetration resistance of the side edges of bucket $W_{y 3}$, friction resistance of prism to the inner and outer side of the plates $W_{y 4}$, vertical load of the prisms on the bucket bottom $W_{y 5}$.

The slippage resistance $W_{y l}$ of the affected material's prisms on the frontal plane of massif:

$$
W_{y l}=g \rho_{z}\left[b_{4}+x \operatorname{tg} \varphi_{o} \cdot \operatorname{tg}\left(\frac{\pi}{4}-\frac{\rho}{2}\right)\right] \frac{\operatorname{tg} \varphi_{o} \sin ^{2} \varphi_{o}}{2} x_{1}^{2}
$$

The slippage resistance $W_{y 2}$ of affected material prism to the vertical side of massif:

$$
W_{y 2}=g \rho_{z} \frac{\operatorname{tg}^{3} \varphi_{o}}{6\left(\operatorname{tg} \varphi_{o}+\operatorname{tg} \beta\right)}\left(1+\sin ^{2} \varphi_{o}\right) \cdot \operatorname{tg}\left(\frac{\pi}{4}-\frac{\rho}{2}\right) x_{1}^{3}
$$
bucket:

The penetration resistance $W_{y 3}$ of the side edges of

$$
W_{y 3}=\frac{W_{x 2}}{\operatorname{tg} \beta}
$$

The friction resistance $W_{y 4}$ of prism to the inner and outer side of the plates:

$$
W_{y 4}=W_{x 4}
$$

The total vertical load of the affected material's prism on bottom of the bucket:

$$
W_{y 5}=\frac{W_{x 3}}{\mu_{2}}
$$

The total vertical resistance of bucket filling:

$$
W_{y}=W_{y 1}+2 W_{y 2}+2 W_{y 3}+4 W_{y 4}+W_{y 5}
$$

\section{PROGRAM}

Based on predefined mathematical model a program (Fig. 4), was developed which provides a detailed kinematic and dynamic analysis of the wheel loader, by simulation of given manipulative tasks.

As an example, using the developed software, kinematic and dynamic analysis of kinematic chain members of wheel loaders with Z-bar manipulator, was performed, mass of loader is $15000 \mathrm{~kg}$ and a volume of bucket is $2.7 \mathrm{~m}^{3}$.

Simulated manipulative task, consisted of next operations: loading of material, lifting the affected material and unloading at a given height.

In this paper, as part of a kinematic analysis, there are given diagrams (Fig. 7) of changes of next values: generalized coordinates $x_{1}, \theta_{3}, \theta_{4}$ (Fig. 7a) of the kinematic chain of loader, the components of linear velocity $v_{4 x}, v_{4 y}$ (sl.7b) and angular velocity $\omega_{4 z}$ and linear acceleration components $a_{4 x}, a_{4 y}(\mathrm{sl} .7 \mathrm{c})$ and angular acceleration $\varepsilon_{4}$ of bucket.

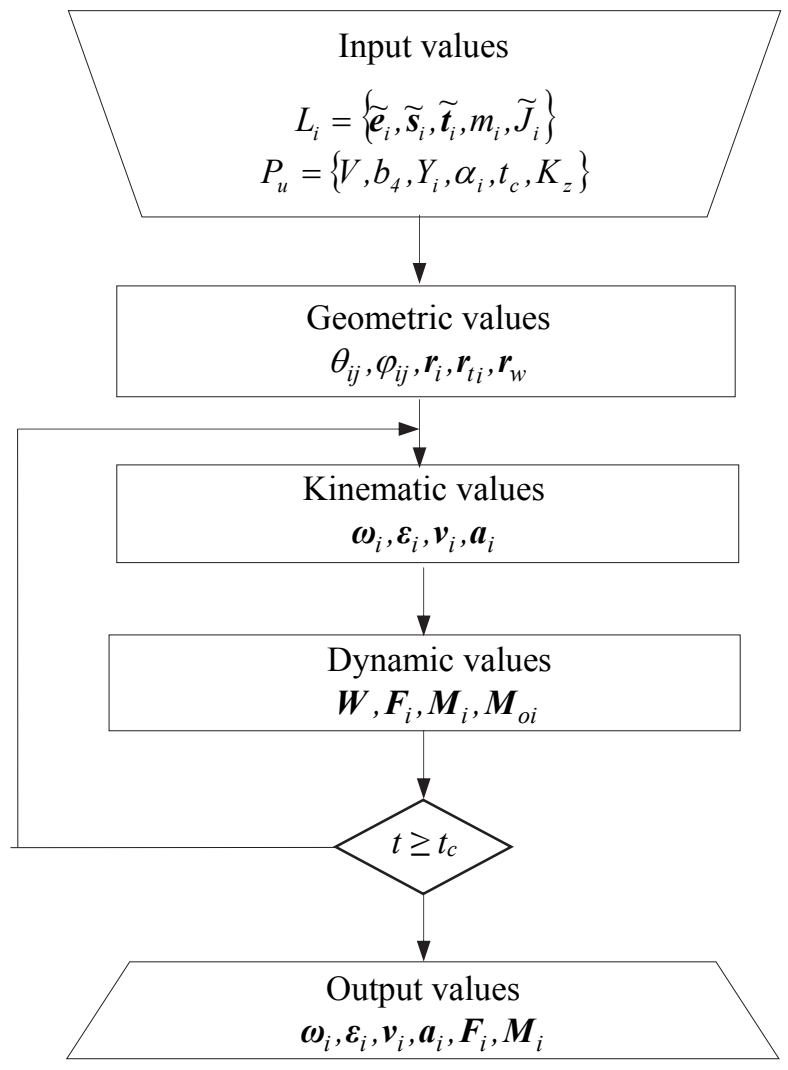

Fig.5. Algorithm of developed program for kinematic and dynamic analysis of the wheel loader 
The changes of generalized coordinates of kinematic chain members (Fig. 6a) show that at loading it comes to simultaneous movements of the moving mechanism members $L_{1}$ and $L_{2}$ and the bucket rotation $L_{4}$. The operation of lifting a loaded material on given height is accomplished by movement of arm $L_{3}$ and depending on movement of bucket $L_{4}$. Bucket rotation, while arm is lifting, occurs because the drive mechanism of bucket is dependent on drive mechanism of arm with aim to maintain the bucket in a position where there is no spilling materials when arm is lifting. Unloading operations are carried out only by running buckets.
Analysis of changes of the speed and acceleration shows that the largest changes in angular velocity $\omega_{4 z}$ (Fig. $6 \mathrm{~b}$ ) and angular acceleration $\varepsilon_{4 z}$ (Fig. 6c) has the bucket at the beginning and at the end of lifting operation, when comes to correction of the bucket that no spilling of materials.

As part of the dynamic analysis there are given diagrams (Fig. 7) of the next changes of values: components of inertial forces of bucket $F_{4 x}, F_{4 y}$ (Fig. 7) and the affected material $F_{z x}, F_{z y}$, inertial torques of arm $M_{3}$ (Fig.7b), bucket $M_{4}$ and affected materials $M_{z}$, static and dynamic load torque of the arm drive mechanism $M_{o 3 s}, M_{o 3}(\mathrm{sl} .7 \mathrm{c})$ and static and
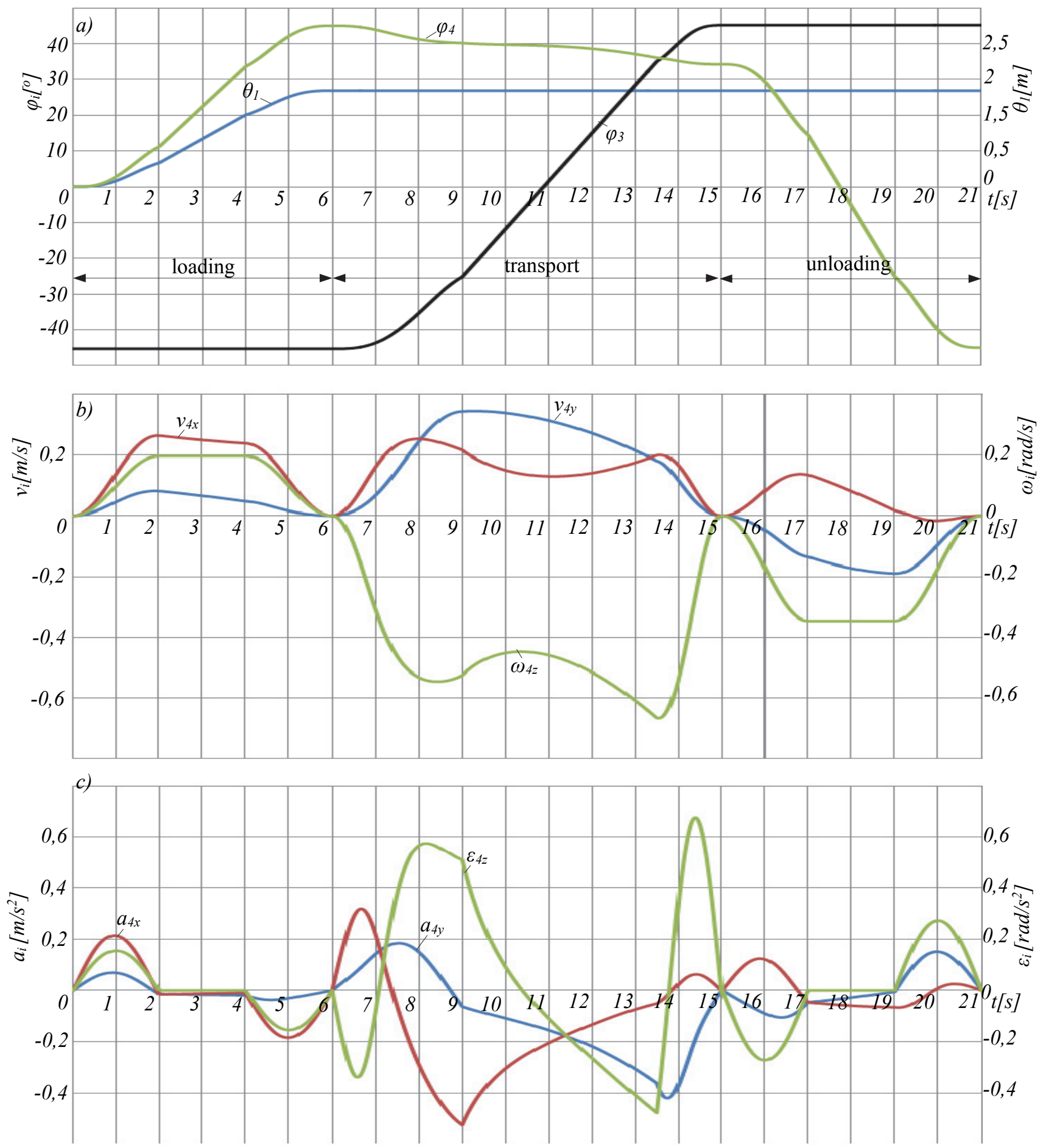

Fig.6. Kinematic analysis of wheel loader: a) change of generalized coordinates, b) components of linear $v_{4}$ and angular velocity $\left.\omega_{4}, c\right)$ components of linear $a_{4}$ and angular accelerations $\varepsilon_{4}$ 

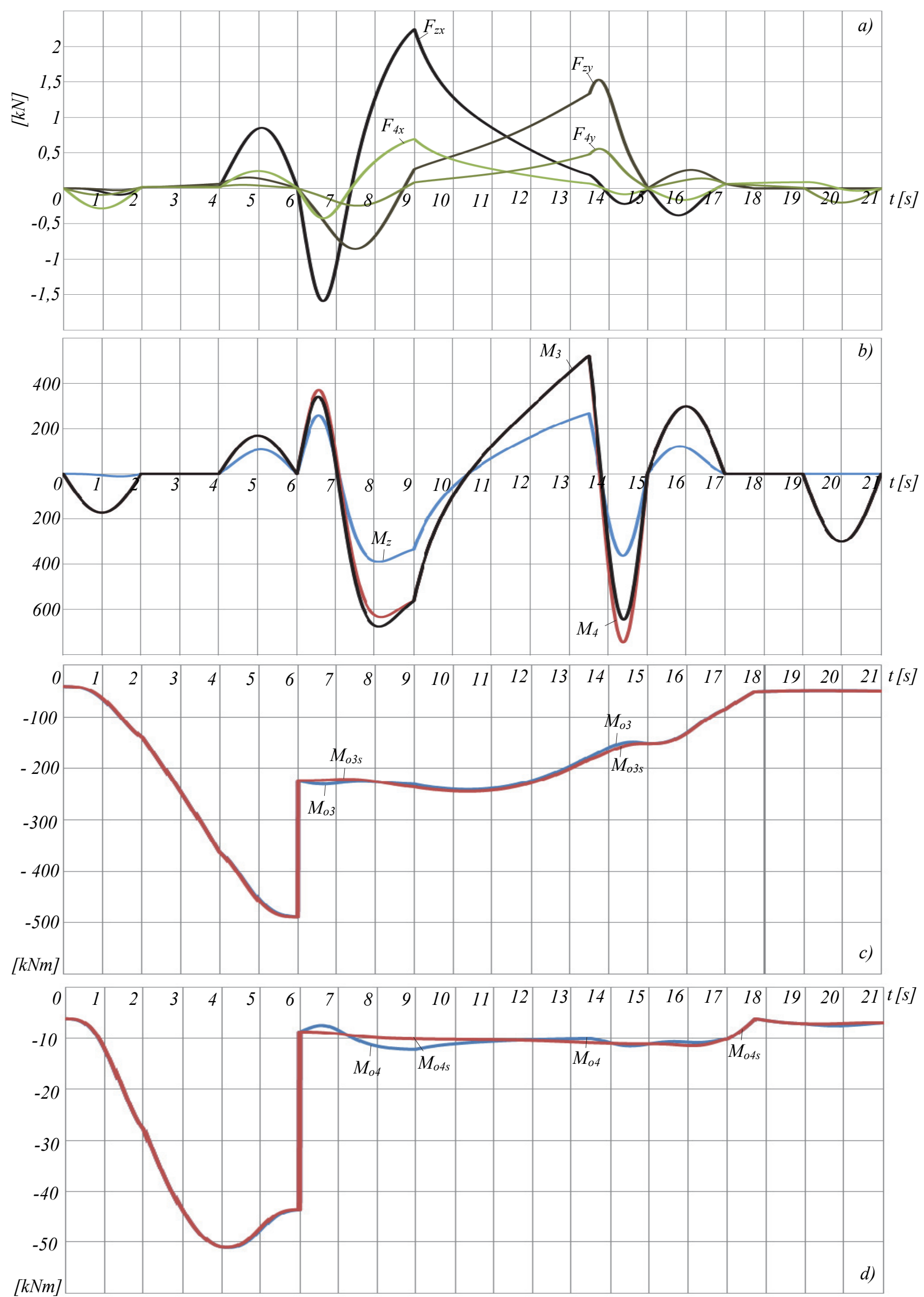

Fig.7. Dynamic analysis of wheel loader: a) change of inertial force, b) change of rotational inertia torque components, $c, d)$ static and dynamic load torques of drive mechanism of arm and bucket 
dynamic load torque of drive mechanism of bucket $M_{o 4 s}, M_{o 4}$ (Fig.7d).

To sudden changes of components of inertial forces of the bucket and affected material occurs at the beginning and end of the transport operation of the affected material. At the beginning of transport operations of material, at lifting of the arm manipulator, the horizontal component of the force of inertia of the affected material has the highest value of variable directions (Fig. 7a). At the end of the operation as the largest force, occurs the vertical component of the inertial force of the affected material (Fig. 7a). Minor changes in inertial forces generated at the end of the operation of loading and at the beginning of the operation of unloading the bucket due to changes in the masses of the affected material.

Extremely variables of the inertial torque of arm, bucket and affected materials also occur at the beginning and end of the operation of the material transport, and the biggest occurring inertial torques are inertial torques of arm and bucket (Fig. 7b).

The analysis of torque of drive mechanism of arm and bucket shows that the largest load torque of mechanisms occur during the operation of materials loading when the difference of static and dynamic load torque is negligible, knowing that the process of loading of material is comparatively slow. Substantial differences of static and dynamic torque load drive mechanism occurs at the beginning of the transport operation and at the operation of unloading of materials due to sudden changes in the mass of the affected material.

\section{CONCLUSION}

In the development of wheel loaders is necessary kinematic and dynamic analysis of kinematic chain members and drive mechanisms of the machine. For these reasons, here are developed a mathematical model and program for detailed kinematic and dynamic analysis of all members of the kinematic chain during simulated manipulative task which consists of operations: loading, transport and unloading of materials. Mathematical model is based on Newton-Euler dynamic equations.

The developed model and software enable that on given sets of manipulative task we can defined: linear and angular velocity and acceleration, the inertial forces and inertia torques of the center of mass of each member of kinematic chains and load torque of drive mechanism of the machine. Kinematic and dynamic parameters that are obtained by simulation presents the basis for defining the structure of kinematic chain members and optimal synthesis of drive mechanism of loader.

\section{ACKNOWLEDGEMENT}

This paper is result of technological project No. TR35049, supported by Ministry of Education, Science and Technological Development of the Republic of Serbia

\section{REFERENCES}

[1] Janošević, D. "Projektovanje mobilnih mašina", Faculty of Mechanical Engineering University of Niš, 2006.

[2] Dresig, H. and Holzweissig, F. "Dynamics of machinery", Springer, Berlin, London, 2010.

[3] Potkonjak, V. "Robotika", Naučna knjiga, Belgrade, 1989.

[4] Brach, I. and Tyro G. "Maszyny ciagnikowe do robot ziemnych", Wydawnietwa Naukowo - Techniczno, Warszawa, 1986.

[5] Jovanović, V., Janošević, D., and Pavlović J. "Experimental determination of resistance digging of hydraulic excavator", IMK-14-Research \& Developement in Heavy Machinery 19(3), pp. 83-88, 2013.

[6] Shen ,J., Wang, G., Bi, Q., and J. Qu “A comprehensive genetic algorithm for design optimization of Z-bar loader working mechanism". Journal of Mechanical Science and Technology Vol.11, No.27, pp. 3381-3394, june 2013.

[7] Pavlović, J., Jovanović M., and Milojević, A. "Optimal synthesis of the manipulator using two competitive methods" Facta universitatis Series: Mechanical Engineering Vol. 12, No 1, pp. 61 - 72, 2014.

[8] Pavlović, J., Janošević, D., Jovanović, V., Milić, P. "Dynamic analysis of the Z-bar loader working mechanism" in proc. The Fifth International Conference TRANSPORT AND LOGISTICS TIL 2014, pp. 119-123, Niš, 2014.

[9] Filla, R. "Quantifying Operability of Working Machines", Linkoping Studies in Science and Technology Dissertations, No 1390, Linkoping 2011.

[10] Filla, R. and Palmberg, J. O. "Using Dynamic Simulation in the Development of Construction Machinery“. The Eighth Scandinavian International Conference on Fluid Power, Tamper, Finland, Vol.1, pp. 651-667, May 7-9, 2003.

[11] Filla, R. "An Event-driven Operator Model for Dynamic Simulation of Construction Machinery". The Ninth Scandinavian International Conference on Fluid Power, Linkoping, Sweden, June 1-3, 2005.

[12] Worley, M. D. and La Saponara, V. "A simplified dynamic model for front-end loader design" Proc. IMechE Vol. 222 Part C: J. Mechanical Engineering Science, SAGE, pp. 2231-2249, 2008. 